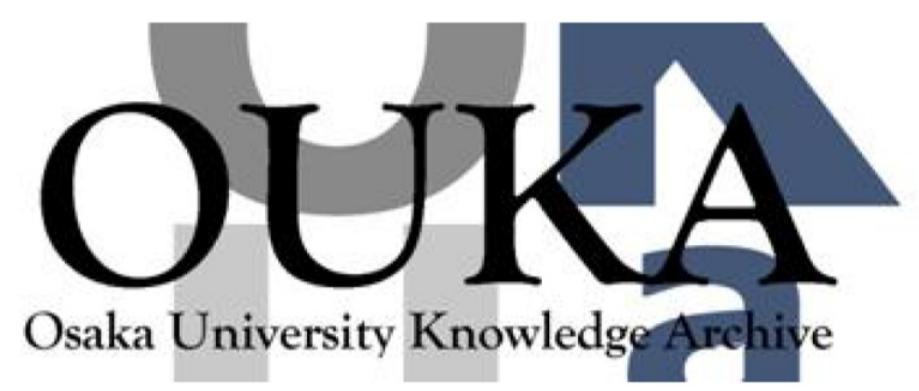

\begin{tabular}{|c|l|}
\hline Title & $\begin{array}{l}\text { Time Passes Slowly When You Are Concealing } \\
\text { Something }\end{array}$ \\
\hline Author(s) & $\begin{array}{l}\text { Matsuda, Izumi; Matsumoto, Ayano; Nittono, } \\
\text { Hiroshi }\end{array}$ \\
\hline Citation & Biological Psychology. 155 p. 107932 \\
\hline Issue Date & $2020-07-22$ \\
\hline oaire:version & AM \\
\hline URL & https://hdl. handle. net/11094/78346 \\
\hline rights & $\begin{array}{l}\text { ○ 2020. This manuscript version is made } \\
\text { avai lable under the CC-BY-NC-ND 4.0 License. }\end{array}$ \\
\hline Note & \\
\hline
\end{tabular}

Osaka University Knowledge Archive : OUKA

https://ir. Library. osaka-u. ac. jp/

Osaka University 


\title{
Time Passes Slowly When You Are Concealing Something
}

\author{
Izumi Matsuda ${ }^{\mathrm{a}}$, Ayano Matsumoto ${ }^{\mathrm{b}}$ and Hiroshi Nittono $\mathrm{b}^{\mathrm{b}^{*}}$
}

aDepartment of Psychology, College of Education, Psychology, and Human Studies, Aoyama Gakuin University, Tokyo, Japan

${ }^{\mathrm{b}}$ Graduate School of Human Sciences, Osaka University, Suita, Japan

ORCiDs:

0000-0002-5626-6751 (Izumi Matsuda)

0000-0001-5671-609X (Hiroshi Nittono)

AM and HN conceived the study; AM conducted the experiment; IM and AM analyzed the data; IM drafted the paper; HN finalized the paper.

Correspondence concerning this article should be addressed to Hiroshi Nittono, Graduate School of Human Sciences, Osaka University, Suita 565-0871, Japan. E-mail: nittono@hus.osaka-u.ac.jp

Please cite the final version:

Matsuda, I., Matsumoto, A., \& Nittono, H. (2020). Time passes slowly when you are concealing something. Biological Psychology, 155, 107932. https:// doi.org/10.1016/j.biopsycho.2020.107932 


\title{
Highlights
}

- Time perception during concealment was investigated.

- People perceived the duration of items as longer when they concealed one of them.

- Skin conductance level was higher in the guilty than in the innocent condition.

- The effort to conceal something leads to a non-specific temporal overestimation.

\begin{abstract}
The item to be concealed elicits greater physiological arousal than other items. Since high physiological arousal causes an overestimation of time, the display duration of an item is expected to be perceived as longer when people intend to conceal it. After stealing and concealing one item, 36 university students were asked to judge the display duration of an item as shorter than, equal to, or longer than a memorised duration of $2 \mathrm{~s}$. Pictures of three items including the stolen item were presented in the guilty condition, whereas pictures of three items that had not been stolen were presented in the innocent condition. The display of all items in the guilty condition was perceived as longer than in the innocent condition without difference between the concealed and other items. The intention to conceal increases tonic arousal reflected in a higher skin conductance level and leads to a non-specific temporal overestimation.
\end{abstract}

Keywords: arousal; concealed information test; skin conductance; time perception; withdrawal motivation 
Imagine a situation in which you must conceal something from people around you. Such a situation will make you nervous, and you will try to keep away from it. Previous studies have shown that an item to be concealed evokes physiological arousal. The concealed information test (CIT), also called the guilty knowledge test, is a test used to evaluate this situation (Verschuere et al., 2011). In this test, a crime-relevant item is presented amid similar items that are not related to the crime while physiological responses are recorded. The items are selected so that innocent examinees cannot identify which item is critical. When greater physiological responses are observed for the crimerelevant item than for the irrelevant items, the examiner infers that the examinee possesses crime-relevant information. Skin conductance, which reflects sympathetic arousal, is most typically measured in the CIT. The crime-relevant item usually elicits a greater skin conductance response (SCR) than the irrelevant items (Meijer et al., 2014).

Some studies have suggested that the intention to conceal recruits withdrawal motivation. When guilty examinees are forced to choose either a crime-relevant or an irrelevant item, they tend to avoid the relevant item (Meijer et al., 2007; Orthey et al., 2018). Moreover, withdrawal motivation may be related to the inhibition of physiological arousal that the recognition of a concealed item initially evokes. This arousal inhibition process is thought to be reflected by heart rate decrease and respiration suppression in the CIT (klein Selle et al., 2016, 2017). When event-related brain potentials are recorded, the concealed item induces right prefrontal cortical activation (Matsuda \& Nittono, 2015b, 2018), which has been linked to the involvement of withdrawal (vs approach) motivational system (Harmon-Jones et al., 2010; Sutton \& Davidson, 1997).

Both physiological arousal and withdrawal motivation are known to affect time perception. Arousal is a key variable in time perception; it accelerates a pacemaker of the internal clock system (Gibbon et al., 1984; Zakay \& Block, 1997). Mella et al. (2011) used a duration comparison task in which participants memorised a standard duration of $2 \mathrm{~s}$ (not explicitly stated) and then judged the duration of each auditory stimulus as shorter than, equal to, or longer than the standard duration, although all the test stimuli lasted 2 s. Participants' SCRs were measured to evaluate phasic physiological arousal. The results showed that highly negative stimuli were perceived to last longer and elicited greater SCRs than less negative and neutral stimuli. Gil and Droit-Volet (2012) used a verbal estimation task in which participants rated the temporal duration of a stimulus in milliseconds. They found that emotion-arousing stimuli were perceived as longer than neutral stimuli, particularly in brief durations $(<1 \mathrm{~s})$. For longer durations, the emotional lengthening effect was reduced because attention was distracted away from the negative stimulus. The content of the pictures also affected time perception; disgust-inducing pictures were judged to be displayed longer than fear-inducing pictures. Therefore, arousal and attention interactively influence time perception depending on the stimulus content and duration (Droit-Volet \& Gil, 2009; Lake et al., 2016). Moreover, not only looking at a negative stimulus but also expecting it leads to an overestimation of time (Vallet et al., 2019), which suggests that sustained emotional states can affect time perception.

Motivational direction has also been suggested to affect time perception. Gable \& Poole (2012) and Gable et al. (2016) used a temporal bisection task in which pictures were displayed for one of seven durations: two standard (short: $400 \mathrm{~ms}$, long: 1,600 ms) and five intermediate durations (600, 800, 1,000, 1,200, and 1,400 ms). Participants were asked to judge whether each picture was displayed for a short or a long period. Positive 
pictures with high approach motivation were more likely to be judged as shorter than positive pictures with low approach motivation or non-emotional pictures (Gable \& Poole, 2012). According to the original authors, this disposition can be biologically adaptive because organisms will readily engage in a goal pursuit for a longer period when they lost track of time. In contrast, overly disgusting images, which induced a strong withdrawalmotivated negative affect, caused a perceived lengthening of temporal duration compared with less disgusting or neutral images (Gable et al., 2016).

In this study, we examined whether the act of concealing something, which is associated with strong physiological arousal and withdrawal motivation, would lead to an overestimation of temporal duration. The duration comparison task of Mella et al. (2011) was combined with the CIT paradigm, where time perception and physiological arousal were recorded simultaneously. At the end of the experiment, participants rated their subjective withdrawal motivation towards each stimulus item. As a control, the same participants were also involved in the innocent condition, in which they did not know the crime-relevant item and thus had nothing to conceal. We tested two hypotheses. First, the display of the concealed crime-relevant item would be perceived to last longer than that of the irrelevant items because it would lead to stronger physiological arousal and withdrawal motivation. Second, the items in the guilty condition would be perceived to be displayed longer than the items in the innocent condition because the effort to conceal something would increase the levels of tonic arousal and withdrawal motivation throughout the task.

\section{Participants}

\section{Method}

Thirty-six graduate and undergraduate students (13 male and 23 female; $20-25$ years old, $M=21.4$ ) volunteered to participate in the experiment. The sample size was determined by a prior power analysis so that a medium effect size $(d z=0.5)$ could be detected with a power of .90 by a one-tailed $t$ test (considering that the hypotheses were directional) or with a power of .83 by a two-tailed $t$ test (see Ogawa and Nittono, 2019 for a similar case). In the following analysis, two-tailed tests were used consistently. The study was approved by the Behavioural Research Ethics Committee of the Osaka University School of Human Sciences. All participants provided written informed consent. The participants received a cash voucher of 500 Japanese yen (equivalent to approximately US \$4.50) at the beginning of the experiment and were told that they could keep it if they successfully followed the concealment instructions until the end of the experiment. After the experiment, the participants were debriefed and received an apology for the deceptive procedure. No cash voucher was retrieved. All participants consented to the use of their data in the analysis. No participants were excluded from the analysis.

\section{Stimuli}

Two sets of real objects were prepared. One set consisted of three accessories (a ring, a necklace, and earrings), and the other consisted of three electronic products (a mobile phone, a digital camera, and a voice recorder). A photograph of each object was taken and used in three angles (upright, left-rotated, and right-rotated), producing 18 pictures in total ( 2 sets $\times 3$ items $\times 3$ angles). The pictures were displayed on an LCD screen (VIEWPixx, VPixx Technologies Inc., Canada) with a visual angle of 10 degrees. The viewing distance was $70 \mathrm{~cm}$. All the stimulus materials and data are available at https://osf.io/9tdej/. 


\section{Procedure}

The participants were told to steal one of six objects (i.e. ring, necklace, earrings, mobile phone, digital camera, or voice recorder) from a drawer in the experimenter's absence and conceal it until the end of the experiment. The picture set that included the stolen item was presented in the guilty condition, while the other set was presented in the innocent condition. The item to be concealed was ostensibly determined by lottery but was in reality assigned in a counterbalanced order by a design unbeknownst to the participants. The stolen item was regarded as the relevant item in the guilty condition. The "relevant" item in the innocent conditions was decided in advance in a counterbalanced order. Although the relevant-item probability of .33 was larger than that used in the typical CIT (e.g. .20), it is still acceptable in the CIT research (Meijer et al., 2014).

Time perception was assessed by a duration comparison task used by Mella et al. (2011). First, the participants memorised the standard duration of 2 s by looking at pictures that would not be used in the main experiment (i.e., three stationaries) nine times. The duration was not explicitly stated. Then, the same pictures were presented for 1.8, 2.0, or $2.2 \mathrm{~s}$. The participants decided whether the duration was "short," "equal," or "long” compared with the memorised standard duration by pressing 4, 5, or 6, respectively, on a numeric keypad. The training phase was completed when the correct response rate exceeded $80 \%$. In the test phase, nine pictures of accessories or electronic products were presented three times in the guilty and innocent conditions. The order of conditions was counterbalanced across participants. Figure 1 illustrates a sequence of events during one trial in the test phase. Each picture appeared on the screen along with the question, "Did you steal this?” All the pictures were presented for $2 \mathrm{~s}$, except for filler trials. In the filler trials, each of the three items (only upright pictures) was presented for 1 or $4 \mathrm{~s}$. The participants made a three-choice assessment of the stimulus duration by pressing a button. Then they orally answered "no" to the question and press the enter key every time in both the guilty and the innocent condition. Oral answers were included to remind the participants of the deception task. No speeded response was required at each step. The next picture appeared on the screen 5-6 s after pressing the enter key. In each condition, 27 test trials ( 3 items $\times 3$ angles $\times 3$ times) and 6 filler trials ( 3 trials each for the 1 - and 4-s durations) were randomly presented. The task was controlled by PsychoPy 3.0 (Peirce et al., 2019). The purpose of the filler trials was to monitor the participants' performance. If a participant conducted the task seriously, the stimuli in the filler trials should be judged as short or long, respectively.

Skin conductance was recorded continuously during the task using a Biopac Student Lab System MP36 (BIOPAC Systems, Inc., CA, USA). Two Ag/AgCl electrodes filled with $0.05 \mathrm{M} \mathrm{NaCl}$ paste (BIOPAC GEL101) were placed on the volar side of the distal phalanges of the index and middle fingers of the non-dominant hand (the left hand in all but one participant). The sampling rate was $15.6 \mathrm{~Hz}$, and no high-pass filter was used. At the end of the experiment, the participants answered three questions about their feelings when they looked at each picture on a 7-point scale: (1) perception of time (1: felt longer, 7: felt shorter), (2) motivational direction (1: want to avoid; 7: want to approach), and (3) stimulus valence (1: unpleasant, 7: pleasant).

\section{Analysis}

The numbers of the "short," "equal," and "long” responses were counted separately for the relevant and irrelevant items in each condition and the mean index of time 
judgement was calculated as (number of "long" responses - number of "short" responses) / total number of responses (Mella et al., 2011). The index ranges from - 1 to 1 . A positive value indicates overestimation, and a negative value indicates underestimation of temporal duration. The interval between the offset of a picture and a button press was recorded as a response time, although no speeded response was required.

The skin conductance level was computed as the mean skin conductance of the entire task duration in each condition. The SCR was calculated as the baseline-to-peak amplitude of the highest peak occurring between 0.5 and $5.0 \mathrm{~s}$ after picture onset. Values smaller than $0.05 \mu \mathrm{S}$ were scored as 0 (Boucsein et al., 2012). Natural log-transformation $(\ln [\mathrm{SCR}(\mu \mathrm{S})+1])$ was used to normalise the amplitude data. In this study, the mean onset-to-onset stimulus interval between consecutive item pictures was about $11 \mathrm{~s}$. Although the intervals were shorter than those in the traditional CIT (e.g., 20 s) and may cause a reduction of absolute SCR amplitudes, differential SCRs between relevant and irrelevant items were expected to be detected, if any (Breska et al., 2011).

The time judgement index, response time, SCR, and subjective ratings (perception of time, motivational direction, and stimulus valence) were subjected to a Condition (guilty or innocent) $\times$ Item (relevant or irrelevant) analysis of variance (ANOVA) with repeated measures. The skin conductance level was compared between the guilty and innocent conditions using a two-tailed $t$ test. The effect sizes were determined using partial $\eta^{2}\left(\eta_{\mathrm{p}}{ }^{2}\right)$ for ANOVAs and Cohen's $d$ for $t$ tests. Cohen's $d$ was computed by dividing the mean difference between the relevant and irrelevant items by the average of the standard deviations of the two items (Lakens, 2013). When testing the difference between two means by a $t$ test, Bayes factor $\left(\mathrm{BF}_{10}\right)$, the odds ratio of the alternative hypothesis $(\mathrm{A} \neq \mathrm{B})$ to the null hypothesis $(\mathrm{A}=\mathrm{B})$, was computed using JASP 0.13 (JASP Team, 2020). A Cauchy distribution with a default scale of 0.707 was used as a prior. $\mathrm{BF}_{10}$ greater than 1 means that the alternative hypothesis is supported over the null hypothesis; $\mathrm{BF}_{10}$ less than 1 gives no evidence for the alternative hypothesis. According the recommendations (Kass \& Raftery, 1995; Wagenmakers et al., 2018), BF 10 greater than 3 was taken as moderate evidence for the alternative model over the null model, whereas $\mathrm{BF}_{10}$ less than $1 / 3$ was taken as moderate evidence for the null model over the alternative model. The BF of the Condition $\times$ Item interaction effect was assessed by a contrast test in which the relevant - irrelevant differences were compared between the guilty and innocent conditions using a paired $t$ test.

\section{Results}

All participants performed the duration comparison task seriously. Thirty-five of them answered perfectly to the 1-s and 4-s filler trials by "short" and "long" responses, respectively; only one participant failed in 2 trials out of the 12 filler trials. This means that they conducted the task seriously and did not press a button randomly.

Figure 2A shows the time judgement index for each condition. The Condition $\times$ Item ANOVA showed a significant main effect of Condition, $F(1,35)=6.85, p=.013, \eta_{\mathrm{p}}{ }^{2}$ $=.16$. The display of items was perceived as longer in the guilty $(M=-0.22)$ than in the innocent condition $(M=-0.31)$. The Bayes factor $\left(\mathrm{BF}_{10}\right)$ was 3.39 , which provided moderate evidence for the alternative hypothesis over the null hypothesis. In contrast, the main effect of Item and the interaction were not significant, $F s(1,35)=3.25$ and 0.76 , $p s$ $=.080$ and $.391, \eta_{\mathrm{ps}}{ }^{2}=.09$ and .02 . The mean differences [95\% confidence intervals] between the relevant and irrelevant items were $-0.08[-0.18,0.01]$ and $-0.04[-0.11$, 
0.03 ] in the guilty and innocent conditions, respectively, which means that the display of the concealed item was not perceived as longer than that of the other items. Bayesian paired $t$ tests also showed no evidence for the differences in time perception of the two items, $\mathrm{BFs}_{10}=0.69$ and 0.30 in the guilty and innocent conditions, respectively. The results were in favour of the null hypothesis. For the interaction contrast, $\mathrm{BF}_{10}$ was 0.25 and supported the null hypothesis that the relevant - irrelevant difference did not differ between the conditions.

The mean response times for the duration judgment were $1,472 \mathrm{~ms}[1,268,1,676]$ and $1,424 \mathrm{~ms}[1,242,1,606]$ for the relevant and irrelevant items in the guilty condition, and 1,302 ms [1,150,1,454] and 1,248 ms [1,100,1,396] for the relevant and irrelevant items in the innocent condition. The Condition $\times$ Item ANOVA for the response time revealed a significant main effect of Condition, $F(1,35)=4.94, p=.033, \eta_{\mathrm{p}}{ }^{2}=.12$. This means that response times increased when participants attempted to conceal. However, the $\mathrm{BF}_{10}$ was close to 1 (1.57) and did not provide strong evidence for the difference. The main effect of Item and the interaction were not significant, $F s(1,35)=1.18$ and $0.01, p s$ $=.285$ and $.923, \eta_{\mathrm{ps}}{ }^{2}=.03$ and $<.01$. The $\mathrm{BF}_{10}$ of the interaction contrast was 0.18 and supported the null hypothesis that the relevant - irrelevant difference did not differ between the conditions.

The mean skin conductance level was higher in the guilty $(M=7.12 \mu \mathrm{S}[6.05,8.19])$ than in the innocent condition ( $M=6.67 \mu \mathrm{S}$ [5.53, 7.81]), $t(35)=3.16, p=.003, d=0.14$. The $\mathrm{BF}_{10}$ was 11.19 , which was large enough to support the difference between the conditions. Figure 2B shows the mean values of SCR and subjective ratings. The ANOVA for SCR showed a significant interaction, $F(1,35)=6.79, p=.013, \eta_{\mathrm{p}}{ }^{2}=.16$. The concealed item induced a greater response than the other items in the same block, $t(35)=$ 2.89, $p=.007, d=0.46, \mathrm{BF}_{10}=6.06$. In contrast, no difference was found in the innocent condition, $t(35)=-0.37, p=.716, d=-0.04, \mathrm{BF}_{10}=0.19$. The mean differences in the unit of $\ln (\mu S+1)$ between the relevant and irrelevant items were $0.04[0.01,0.07]$ in the guilty condition and $0.00[-0.02,0.01]$ in the innocent condition. The $\mathrm{BF}_{10}$ of the interaction contrast was 3.31 and supported the alternative hypothesis that the relevant irrelevant difference differed between the conditions.

Table 1 shows the results of the subjective ratings after the experiment. The subjective perception of time did not differ between items or between conditions; neither the main effect of condition nor the interaction was significant, $F(1,35)=0.77, p=.385$, $\eta_{\mathrm{p}}{ }^{2}=.02$ and $F(1,35)=0.02, p=.893, \eta_{\mathrm{p}}{ }^{2}<.01$, respectively. The $\mathrm{BFs}_{10}$ of the main effect and the interaction contrast were 0.26 and 0.18 , respectively, both of which supported the null hypothesis. Regarding motivational direction, a marginally significant interaction was observed, $F(1,35)=4.02, p=.053, \eta_{\mathrm{p}}{ }^{2}=.10$. Although the concealed item was numerically more associated with withdrawal ("want to avoid") than the irrelevant items, the $\mathrm{BF}_{10}$ of the interaction contrast was 1.07, which gave no evidence either for the alternative or for the null hypothesis. There was a significant interaction for stimulus valence, $F(1,35)=7.18, p=.011, \eta_{\mathrm{p}}{ }^{2}=.17$. The concealed items were rated more negatively than the other items in the guilty condition, $t(35)=-3.24, p=.003, d=$ $-0.62, \mathrm{BF}_{10}=13.40$, whereas no difference was found in the innocent condition, $t(35)=$ 0.391, $p=.698, d=0.07, \mathrm{BF}_{10}=0.19$. The $\mathrm{BF}_{10}$ of the interaction contrast was 3.85, which supported the alternative hypothesis that the relevant - irrelevant difference differed between the conditions. 


\section{Discussion}

This study investigated the time perception of concealed items using a duration comparison task (Mella et al., 2011). Given that the presentation of a concealed item elicits stronger physiological arousal and withdrawal motivation than that of irrelevant items, we hypothesised that the display duration of the concealed item would be perceived as longer than that of the other items. As predicted, the SCR was greater for the concealed item than for the irrelevant items. However, time perception did not differ between the concealed and irrelevant items, either behaviourally or subjectively. In contrast, the display of all items in the guilty condition was perceived as longer than that in the innocent condition. The former condition was accompanied by stronger tonic arousal than the latter, which was confirmed by the skin conductance level. Subjective measures did not show the expected results except that the concealed items were rated more negatively than the other items.

Our finding that time perception did not differ between the concealed and irrelevant items appears to contradict the findings of previous studies. A stimulus that elicits stronger physiological arousal and a stronger negative affect should be perceived as longer (Gable et al., 2016; Mella et al., 2011). In our study, there was no statistically significant effect of concealment; rather, the concealed item tended to be judged numerically shorter than the other items. This inconsistency can be explained by the amount of attention allocated to the stimuli. Coull et al. (2004) showed that a stimulus was felt to be shorter when it was less attended, suggesting neural substrates of the phenomenon. Mella et al. (2011) reported that the duration of aversive sounds was perceived as longer when participants were asked to attend more to the emotional intensity of the stimuli. Gil and Droit-Volet (2012) found that the effect of arousal on time perception decreased when the stimulus duration exceeded $1 \mathrm{~s}$ because attention was diverted away from a stimulus with negative valence. Because we used a duration of $2 \mathrm{~s}$, it is likely that the attention diversion effect cancelled out the arousal effect. That is, the relevant item would be perceived to be displayed longer if it was presented for less than $1 \mathrm{~s}$, where the effect of initial phasic arousal would not be masked.

Previous studies have suggested a two-process model of the CIT. Phasic physiological arousal occurs initially when participants identify a relevant item, and if they attempt to conceal it, they will inhibit the physiological arousal (klein Selle et al., 2016, 2017). The former process is reflected in a higher SCR, whereas the latter process is reflected in heart rate deceleration and respiratory suppression. Studies using eventrelated brain potentials have shown that the latter controlled inhibition process is initiated about 500 ms after stimulus onset (Matsuda \& Nittono, 2015a, 2018). In this study, the stimuli to be judged were presented for $2 \mathrm{~s}$, which was longer enough to initiate the inhibition process. The participants may have diverted their attention from the critical item to inhibit their initial arousal response, and this attentional diversion may have cancelled out the temporal overestimation of a highly arousing concealed item (Gil \& Droit-Volet, 2012).

Another possible explanation might be the use of countermeasures. To deceive the experimenter, participants may have tried to equalise the ratio of "short," "equal," and "long" responses between the concealed and the other items. However, this seems difficult to perform because the participants did not know the correct answer (i.e. the exact stimulus duration) for each trial. 
On the other hand, the display of all items in the guilty condition was perceived as longer than that of the same items in the innocent condition. Additionally, response times for duration judgement were longer in the guilty condition than in the innocent condition. Although no speeded response was required, this result may suggest that concealing something was demanding. The skin conductance level showed stronger tonic physiological arousal in the guilty condition. This result is consistent with a recent finding that a greater (but not significant) increase in skin conductance level and significantly higher anticipatory SCRs were observed before participants decided to conceal an item than when they decided not to conceal (i.e., reveal) it (klein Selle et al., 2019). Concealing something produces sustained tension and thus elevates tonic physiological arousal. If tonic arousal speeds up a pacemaker of the internal clock system (Cheng et al., 2016; Gibbon et al., 1984; Zakay \& Block, 1997), it causes an overestimation of time during the task, irrespective of whether the item should be concealed or not. Using a temporal reproduction task, van Hedger et al. (2017) found that positive and negative affective images were experienced as lasting longer under social stress induced by the preparation of delivering a speech in front of a video camera than in the baseline period. This situation involves the fear of evaluation and resembles our study's concealment task, in which the participants' acts were overseen by the examiner. Van Hedger et al., however, reported that the lengthening effect was not observed in non-emotional stimuli. This contradicts our result and might be due to differences in the temporal task used and the duration to be judged (Gil \& Droit-Volet, 2012; Lake et al., 2016).

In summary, this study shows for the first time that the act of deception distorts time perception. This phenomenon corresponds with our intuition, but it has not been tested empirically. When we try to conceal something, the presentation of not only the critical item to be concealed but also other items presented along with it is perceived as lasting longer than usual. This tendency appears to be generated by a heightened tonic arousal level due to deception-related mental effort. Although the concealed item did elicit stronger phasic physiological arousal than the other items, this did not alter the temporal perception of the concealed item exclusively, at least for a longer duration (2 s), which allows top-down processing of arousal inhibition. Further research using a shorter duration or the instruction not to conceal the item will elucidate the whole picture of the relationship between concealment and time perception. Specifically, the question remains whether the critical item would be perceived to be displayed longer, reflecting its initial phasic arousal, when it is presented for less than $1 \mathrm{~s}$ or when the participants do not have to conceal it. 


\section{Acknowledgements}

This study was supported by JSPS KAKENHI Grant Number 17K04513 to Izumi Matsuda. We thank Gershon Ben-Shakhar and Nathalie klein Selle for their helpful comments on the manuscript.

\section{Declaration of interest statement}

The authors declare no conflicts of interest associated with this study.

\section{References}

Boucsein, W., Fowles, D. C., Grimnes, S., Ben-Shakhar, G., Roth, W. T., Dawson, M. E., \& Filion, D. L. (2012). Publication recommendations for electrodermal measurements. Psychophysiology, 49, 1017-1034. https://doi.org/10.1111/j.14698986.2012.01384.X

Breska, A., Maoz, K., \& Ben-Shakhar, G. (2011). Interstimulus intervals for skin conductance response measurement. Psychophysiology, 48, 437-440. https://10.1111/j.1469-8986.2010.01084.x

Cheng, R. K., Tipples, J., Narayanan, N. S., \& Meck, W. H. (2016). Clock speed as a window into dopaminergic control of emotion and time perception. Timing \& Time Perception, 4, 99-122. https://doi.org/10.1163/22134468-00002064

Coull, J. T., Vidal, F., Nazarian, B., \& Macar, F. (2004). Functional anatomy of the attentional modulation of time estimation. Science, 303, 1506-1508. https://doi.org/10.1126/science.1091573

Droit-Volet, S., \& Gil, S. (2009). The time-emotion paradox. Philosophical Transactions of the Royal Society B: Biological Sciences, 364, 1943-1953. https://doi.org/10.1098/rstb.2009.0013

Gable, P. A., Neal, L. B., \& Poole, B. D. (2016). Sadness speeds and disgust drags: Influence of motivational direction on time perception in negative affect. Motivation Science, 2, 238-255. https://doi.org/10.1037/mot0000044

Gable, P. A., \& Poole, B. D. (2012). Time flies when you're having approach-motivated fun: Effects of motivational intensity on time perception. Psychological Science, 23, 879-886. https://doi.org/10.1177/0956797611435817

Gibbon, J., Church, R. M., \& Meck, W. H. (1984). Scalar timing in memory. Annals of the New York Academy of Sciences, 423, 52-77. https://doi.org/10.1111/j.17496632.1984.tb23417.x

Gil, S., \& Droit-Volet, S. (2012). Emotional time distortions: The fundamental role of arousal. Cognition and Emotion, 26, 847-862. https://doi.org/10.1080/02699931.2011.625401

Harmon-Jones, E., Gable, P. A., \& Peterson, C. K. (2010). The role of asymmetric frontal cortical activity in emotion-related phenomena: A review and update. Biological Psychology, 84, 451-462. https://doi.org/10.1016/j.biopsycho.2009.08.010

JASP Team (2020). JASP (version 0.13) [computer software]. https://jasp-stats.org/

Kass, R. E., \& Raftery, A. E. (1995). Bayes factors. Journal of the American Statistical Association, 90, 773-795. https://doi.org/10.2307/2291091

klein Selle, N., Agari, N., \& Ben-Shakhar, G. (2019). Hide or seek? Physiological responses reflect both the decision and the attempt to conceal information. Psychological Science, 30, 1424-1433. https://doi.org/10.1177/0956797619864598 
klein Selle, N., Verschuere, B., Kindt, M., Meijer, E., \& Ben-Shakhar, G. (2016). Orienting versus inhibition in the Concealed Information Test: Different cognitive processes drive different physiological measures. Psychophysiology, 53, 579-590. https://doi.org/10.1111/psyp.12583

klein Selle, N., Verschuere, B., Kindt, M., Meijer, E., \& Ben-Shakhar, G. (2017). Unraveling the roles of orienting and inhibition in the Concealed Information Test. Psychophysiology, 54, 628-639. https://doi.org/10.1111/psyp.12825

Lake, J. I., LaBar, K. S., \& Meck, W. H. (2016). Emotional modulation of interval timing and time perception. Neuroscience \& Biobehavioral Reviews, 64, 403-420. https://doi.org/10.1016/j.neubiorev.2016.03.003

Lakens, D. (2013). Calculating and reporting effect sizes to facilitate cumulative science: A practical primer for t-tests and ANOVAs. Frontiers in Psychology, 4, 863. https://doi.org/10.3389/fpsyg.2013.00863

Matsuda, I., \& Nittono, H. (2015a). Motivational significance and cognitive effort elicit different late positive potentials. Clinical Neurophysiology, 126, 304-313. https://doi.org/10.1016/j.clinph.2014.05.030

Matsuda, I., \& Nittono, H. (2015b). The intention to conceal activates the right prefrontal cortex: An ERP study. NeuroReport, 26, 223-227. https://doi.org/10.1097/WNR.0000000000000332

Matsuda, I., \& Nittono, H. (2018). A concealment-specific frontal negative slow wave is generated from the right prefrontal cortex in the Concealed Information Test. Biological Psychology, 135, 194-203. https://doi.org/https://doi.org/10.1016/j.biopsycho.2018.04.002

Meijer, E. H., klein Selle, N., Elber, L., \& Ben-Shakhar, G. (2014). Memory detection with the Concealed Information Test: A meta analysis of skin conductance, respiration, heart rate, and P300 data. Psychophysiology, 51, 879-904. https://doi.org/10.1111/psyp.12239

Meijer, E. H., Smulders, F. T., Johnston, J. E., \& Merckelbach, H. L. (2007). Combining skin conductance and forced choice in the detection of concealed information. Psychophysiology, 44, 814-822. https://doi.org/10.1111/j.1469-8986.2007.00543.x

Mella, N., Conty, L., \& Pouthas, V. (2011). The role of physiological arousal in time perception: psychophysiological evidence from an emotion regulation paradigm. Brain and Cognition, 75, 182-187. https://doi.org/10.1016/j.bandc.2010.11.012

Ogawa, Y., \& Nittono, H. (2019). The effect of induced mood on word imagery processing: An ERP study. International Journal of Psychophysiology, 142, 17-24. https://doi.org/10.1016/j.ijpsycho.2019.05.010

Orthey, R., Vrij, A., Meijer, E., Leal, S., \& Blank, H. (2018). Resistance to coaching in forced-choice testing. Applied Cognitive Psychology, 32, 693-700. https://doi.org/10.1002/acp.3443

Peirce, J., Gray, J. R., Simpson, S., MacAskill, M., Hochenberger, R., Sogo, H., Kastman, E., \& Lindelov, J. K. (2019). PsychoPy2: Experiments in behavior made easy. Behavior Research Methods, 51, 195-203. https://doi.org/10.3758/s13428-01801193-y

Sutton, S. K., \& Davidson, R. J. (1997). Prefrontal brain asymmetry: A biological substrate of the behavioral approach and inhibition systems. Psychological Science, 8, 204-210. https://doi.org/10.1111/j.1467-9280.1997.tb00413.x 
Vallet, W., Laflamme, V., \& Grondin, S. (2019). An EEG investigation of the mechanisms involved in the perception of time when expecting emotional stimuli. Biological Psychology, 148, 107777. https://doi.org/10.1016/j.biopsycho.2019.107777

van Hedger, K., Necka, E. A., Barakzai, A. K., \& Norman, G. J. (2017). The influence of social stress on time perception and psychophysiological reactivity. Psychophysiology, 54, 706-712. https://doi.org/10.1111/psyp.12836

Verschuere, B., Ben-Shakhar, G., \& Meijer, E. (2011). Memory detection: Theory and application of the concealed information test. Cambridge University Press.

Wagenmakers, E.-J., Love, J., Marsman, M., Jamil, T., Ly, A., Verhagen, J., Selker, R., Gronau, Q. F., Dropmann, D., Boutin, B., Meerhoff, F., Knight, P., Raj, A., van Kesteren, E.-J., van Doorn, J., Šmíra, M., Epskamp, S., Etz, A., Matzke, D., . . . Morey, R. D. (2018). Bayesian inference for psychology. Part II: Example applications with JASP. Psychonomic Bulletin \& Review, 25, 58-76. https://doi.org/10.3758/s13423-017-1323-7

Zakay, D., \& Block, R. A. (1997). Temporal cognition. Current Directions in Psychological Science, 6, 12-16. https://doi.org/10.1111/1467-8721.ep11512604 


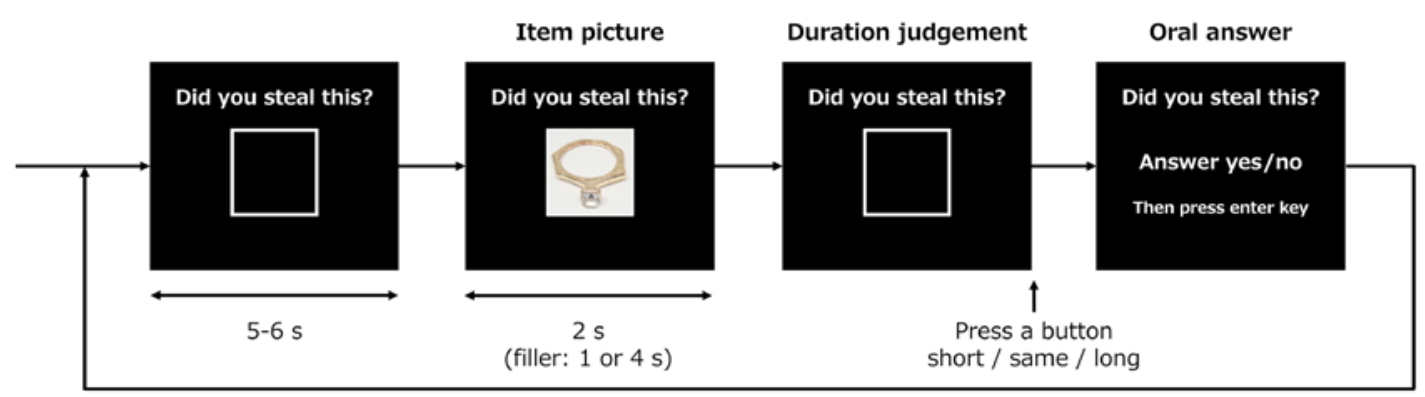

Figure 1. The duration comparison task used in this study. No speeded response was required for either duration judgement or oral answer. 
A

B
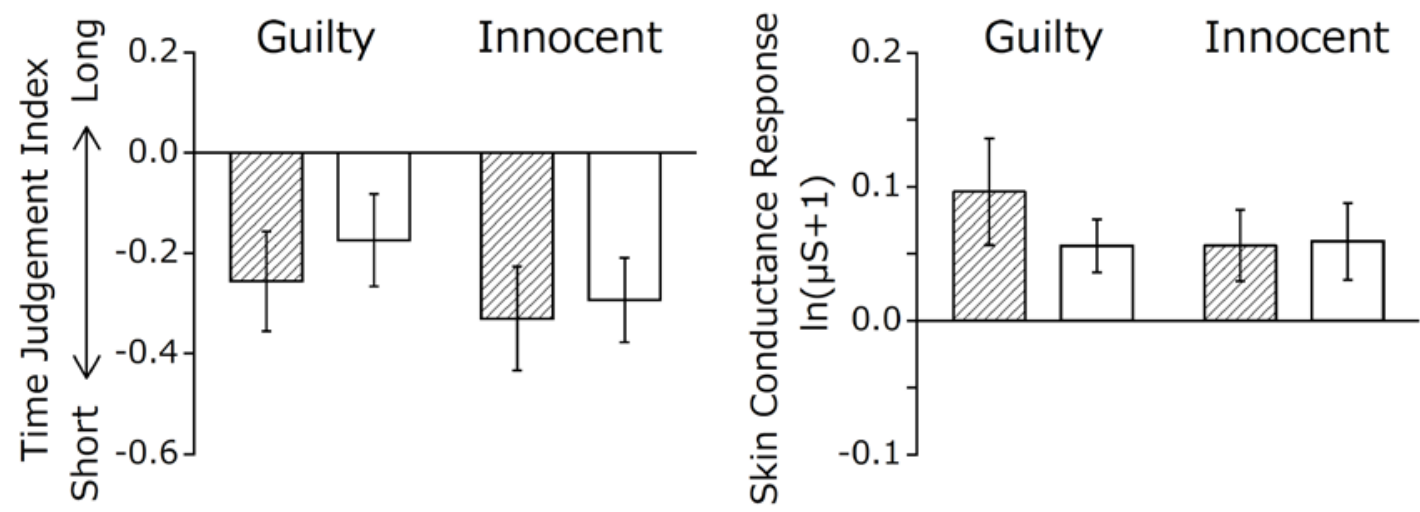

melevant

Irrelevant

Figure 2. The perception of temporal duration (A) and phasic arousal responses (B) for the relevant and irrelevant items in the guilty and innocent conditions. The relevant stimulus in the guilty condition was the item that participants had to conceal. 
Table1. Means and 95\% confidence intervals of the post-experiment subjective reports Perception of time Motivational direction Stimulus valence (1: felt longer, $\quad$ (1: want to avoid, (1: unpleasant, 7: felt shorter) $\quad$ 7: want to approach) $\quad$ 7: pleasant)

\section{Guilty condition}

$\begin{array}{lccc}\text { Relevant (concealed) } & 3.92 & 3.61 & 3.61 \\ & {[3.59,4.24]} & {[3.26,3.97]} & {[3.27,3.95]} \\ \text { Irrelevant } & 3.76 & 3.90 & 4.14 \\ & {[3.52,4.01]} & {[3.66,4.14]} & {[3.90,4.38]} \\ \text { Relevant }- \text { Irrelevant } & 0.15 & -0.29 & -0.53 \\ & {[-0.25,0.56]} & {[-0.59,0.00]} & {[-0.86,-0.20]}\end{array}$

Innocent condition

\begin{tabular}{lccc} 
Relevant & 4.00 & 3.86 & 3.86 \\
& {$[3.62,4.38]$} & {$[3.62,4.11]$} & {$[3.58,4.14]$} \\
Irrelevant & 3.89 & 3.86 & 3.81 \\
& {$[3.64,4.14]$} & {$[3.63,4.09]$} & {$[3.56,4.06]$} \\
\multirow{2}{*}{ Relevant - Irrelevant } & 0.11 & 0.00 & 0.06 \\
& {$[-0.28,0.50]$} & {$[-0.23,0.23]$} & {$[-0.23,0.34]$} \\
\hline
\end{tabular}

